\title{
Communication \\ Frequency-Amplitude Relationship of a Nonlinear Symmetric Panel Absorber Mounted on a Flexible Wall
}

\author{
Yiu-Yin Lee
}

check for updates

Citation: Lee, Y.-Y. Frequency-

Amplitude Relationship of a

Nonlinear Symmetric Panel Absorber Mounted on a Flexible Wall. Symmetry 2021, 13, 1188. https:// doi.org/10.3390/sym13071188

Academic Editors: Giuseppe Grassi and Jan Awrejcewicz

Received: 29 April 2021

Accepted: 2 June 2021

Published: 1 July 2021

Publisher's Note: MDPI stays neutral with regard to jurisdictional claims in published maps and institutional affiliations.

Copyright: (C) 2021 by the author. Licensee MDPI, Basel, Switzerland. This article is an open access article distributed under the terms and conditions of the Creative Commons Attribution (CC BY) license (https:/ / creativecommons.org/licenses/by/ $4.0 /)$.
Department of Architecture and Civil Engineering, City University of Hong Kong, Tat Chee Avenue, Kowloon 852, Hong Kong; bcraylee@cityu.edu.hk

\begin{abstract}
This study addresses the frequency-amplitude relationship of a nonlinear symmetric panel absorber mounted on a flexible wall. In many structural-acoustic works, only one flexible panel is considered in their models with symmetric configuration. There are very limited research investigations that focus on two flexible panels coupled with a cavity, particularly for nonlinear structural-acoustic problems. In practice, panel absorbers with symmetric configurations are common and usually mounted on a flexible wall. Thus, it should not be assumed that the wall is rigid. This study is the first work employing the weighted residual elliptic integral method for solving this problem, which involves the nonlinear multi-mode governing equations of two flexible panels coupled with a cavity. The reason for adopting the proposed solution method is that fewer nonlinear algebraic equations are generated. The results obtained from the proposed method and finite element method agree reasonably well with each other. The effects of some parameters such as vibration amplitude, cavity depth and thickness ratio, etc. are also investigated.
\end{abstract}

Keywords: nonlinear oscillation; panel absorber; frequency-amplitude formulation; harmonic balance

\section{Introduction}

Panel absorbers are used to control sound reflection, absorption, echo and reverberation in a room. Typically, they are made of thin metal or plastic sheets backed by a symmetrical air gap. These thin panel absorbers vibrate nonlinearly. Common linear designs for nonlinearly vibrating structure are inappropriate and can result in overdesign. Otherwise, their hardware configurations are quite symmetric. Usually, there are no complicated geometrical boundary conditions and features. Therefore, classical or analytical methods are more suitable than finite element methods for modelling and theoretical development. Due to their symmetric properties, the modal coupling effects between the mode shapes of a panel absorber are significant. Although considerable research on panel absorbers has been conducted (e.g., [1-9]), most of this research assumed that (1) the panel absorber was mounted on a rigid wall, and (2) the panel vibration was linear. Note that, in practice, panel absorbers are usually made of a thin sheet and mounted on a flexible wall. Previously, the forced vibration of a panel-cavity system with two flexible panels in [10] was studied using the harmonic balance method. However, frequency-amplitude analysis was not included in [10] or in any other related works. In the work of [11], two governing equations for two identical panels with same vibration amplitude coupled with a cavity were simplified to become just one governing equation. This is the equivalent of a governing equation for a panel-cavity problem with only one flexible panel (not two). Moreover, nonlinear plate vibration and nonlinear oscillation have been popular research topics for many years (e.g., [12-16]). Various harmonic balance methods have been commonly used for nonlinear problems (e.g., [17-20]); however, the classical harmonic method is quite time consuming. When a solution is required to be more accurate, more nonlinear algebraic equations are generated in the harmonic balance process. Thus, the weighted residual elliptic integral method is adopted to study the nonlinear effect on the resonant frequencies 
of the entire vibro-acoustic system. The advantage of the proposed solution method is that the elliptic integral solution form contains all harmonic characters. This results in fewer nonlinear algebraic equations needing to be handled. This study also focuses on the modelling of two flexible panels coupled with a cavity and the corresponding nonlinear formulation development.

\section{Theoretical Formulation (Structural Part)}

Figure 1 shows a flexible wall mounted with a symmetric panel absorber, which consists of a nonlinear panel and a cavity. It is assumed that the panel absorber and flexible wall are simply supported. According to $[10,11]$, the governing equations of the nonlinear symmetric panel and flexible wall are shown in the following,

$$
\begin{gathered}
\rho_{c} \frac{d^{2} A}{d t^{2}}+\rho_{c} \omega_{c}^{2} A+\beta_{c} A^{3}+P_{c}=0 \\
\rho_{o} \frac{d^{2} B}{d t^{2}}+\rho_{o} \omega_{o}^{2} B-P_{o}=0
\end{gathered}
$$

where $A$ and $B$ are the vibration responses of the nonlinear symmetric panel and flexible wall subject to the normalized acoustic pressures $P_{\mathcal{c}}$ and $P_{o}$, respectively. $P_{\mathcal{C}}$ and $P_{o}$ depend on $A$ and $B$. Thus, Equations (1) and (2) are coupled (the derivations of $P_{c}$ and $P_{o}$ are shown in the next section);

$\omega_{c}, \omega_{0}, \ell_{c}$ and $\ell_{0}$ are the linear resonant frequencies and surface densities of the nonlinear symmetric panel and flexible wall, respectively;

$\beta_{c}$ is the nonlinear symmetric panel $\left(=\frac{E \tau}{4\left(1-v^{2}\right)}\left(\frac{m \pi}{a}\right)^{4}\left[\left(1+\left(\frac{n}{m} \gamma\right)^{4}\right)\left(\frac{3}{4}-\frac{v^{2}}{4}\right)+v\left(\frac{n}{m} \gamma\right)^{2}\right]\right)$ $m$ and $n$ are the mode numbers of the panel absorber;

$a$ is the panel width;

$\gamma$ is aspect ratio;

$\tau_{c}$ is panel thickness;

$E$ is Young's modulus;

$v$ is Poisson's ratio;

$t$ is time.

Note that the flexible wall is thick and vibrating linearly. Thus, its governing equation in Equation (2) is also linear.

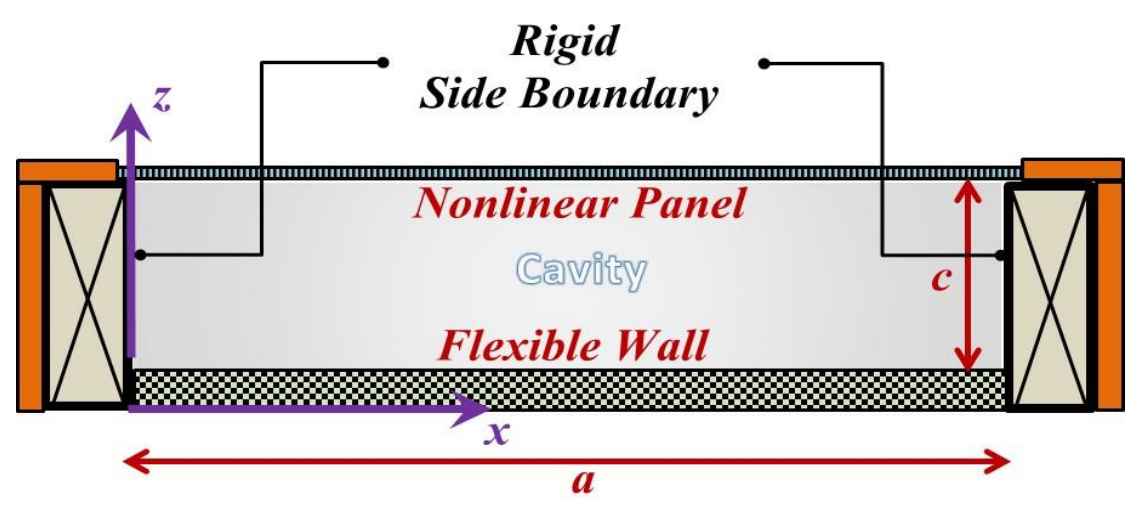

Figure 1. Nonlinear symmetric panel absorber mounted on a flexible wall.

Rewriting Equations (1) and (2) by changing $A$ and $B$ to $\bar{A}$ and $\bar{B}$ gives the following equations

$$
\begin{gathered}
\rho_{c} \frac{d^{2} \bar{A}}{d t^{2}}+\rho_{c} \omega_{c}^{2} \bar{A}+\beta_{c} \bar{A}^{3}+P_{c}=R_{c} \\
\rho_{o} \frac{d^{2} \bar{B}}{d t^{2}}+\rho_{o} \omega_{o}^{2} \bar{B}-P_{o}=R_{o}
\end{gathered}
$$


where

$\bar{A}=A_{\text {in }} C n(\Phi(\kappa))$ is the approximate solution form of the Duffing equation (see below), $\frac{d^{2} \bar{A}}{d t^{2}}+\left(\rho_{c} \omega_{o}^{2}+K_{A}\right) \bar{A}+\beta_{c} \bar{A}^{3}=0 ; \kappa=\frac{\beta_{c} A_{\text {in }}{ }^{2}}{2\left(\rho_{c} \omega_{c}^{2}+K_{A}+\beta_{c} A_{\text {in }}{ }^{2}\right)} ;$

$\Phi$ and $\kappa$ are the elliptical integral and its modulus, respectively;

$A_{\text {in }}$ is the initial vibration amplitude and $K_{A}$ is an unknown constant to be determined;

$\bar{B}=B_{0} \cos (\omega t)$ is the approximate solution form of the linearly vibrating flexible wall;

$B_{0}$ is the vibration amplitude of the flexible wall.

Note that $\bar{A}$ and $\bar{B}$ are not the exact solution forms. Thus, the residual terms, $R_{c}$ and $R_{0}$, are considered on the right side of Equations (3) and (4). $\omega$ is the nonlinear resonant frequency of the whole structural acoustic system and given by [11]

$$
\omega=\frac{2 \pi}{\frac{4}{\left(\omega_{c}^{2}+\frac{K_{A}+\beta A_{\text {in }}{ }^{2}}{\rho_{c}}\right)^{1 / 2}} \int_{0}^{\pi / 2} \frac{1}{\sqrt{1-\kappa^{2} \sin (\Psi)^{2}}} d \Psi}
$$

where $T=2 \pi / \omega$ is the period.

Equations (3) and (4) can be rewritten as

$$
\begin{gathered}
-K_{A} \bar{A}+P_{c}=R_{c} \\
-\rho_{o}\left(\omega^{2}-\omega_{o}^{2}\right) \bar{B}-P_{o}=R_{o}
\end{gathered}
$$

By setting an initial vibration amplitude for the nonlinear symmetric panel and solving the following weighted two residual equations, the unknown constant $K_{A}$ can be found. Then, the nonlinear resonant frequency (i.e., $\omega$ ) can be found using Equation (5)

$$
\begin{aligned}
& \int_{0}^{T} W_{c} R_{c} d t=0 \\
& \int_{0}^{T} W_{o} R_{o} d t=0
\end{aligned}
$$

where $W_{c}$ and $W_{o}$ are the weighting functions. In this study, $W_{c}$ and $W_{o}$ are set as $\cos (\omega t)$. $P_{c}$ and $P_{o}$ depend on $\omega, \bar{A}$ and $\bar{B}$ (or $A_{\text {in }}$ and $B_{o}$ ), and so do the residuals in Equations (6)-(9). Therefore, Equations (8) and (9) are coupled. By assigning a fixed vale to $A_{\text {in }}$ as the initial vibration amplitude, $\omega$ and $B_{o}$ in Equations (8) and (9) can be found using the Mathcad equation solver (i.e., two equations, two unknowns). Note that the derivations of $P_{c}$ and $P_{o}$ are shown in the next section.

\section{Theoretical Formulation (Acoustic Part)}

In this section, the derivations of the two normalized acoustic pressures (i.e., $P_{c}$ and $P_{o}$ in Equations (1) and (2)) are shown and described. Firstly, with reference to the 3-dimensional homogeneous wave equation [10-12,20]:

$$
\nabla^{2} P(x, y, z, t)-\frac{1}{C_{a}^{2}} \frac{\partial^{2} P(x, y, z, t)}{\partial t^{2}}=0
$$

where $C_{a}$ is the speed of sound; $P(x, y, z, t)$ is the acoustic pressure within the cavity at a position of $(x, y, z)$, which can be expressed as a summation of its harmonic components

$$
P(x, y, z, t)=\sum_{h=1,3,5 .}^{H} P^{h}(x, y, z, t)
$$

where $P^{h}$ is the $h$ th harmonic component of $P ; H$ is the number of harmonic components considered. Note that each $P^{h}$ can also satisfy the 3-dimensional homogeneous wave equation. 
The general solution form of the homogeneous wave equation is given by

$$
P^{h}(x, y, z, t)=\sum_{u}^{U} \sum_{v}^{V}\left[L_{u v}^{h} \sinh \left(\mu_{u v}^{h} z\right)+N_{u v}^{h} \cosh \left(\mu_{u v}^{h} z\right)\right] \varphi_{u v}(x, y) \cos (h \omega t)
$$

where $\mu_{u v}^{h}=\sqrt{(u \pi / a)^{2}+(v \pi / b)^{2}-\left(h \omega / c_{a}\right)^{2}}$;

$a$ and $b$ are the length and width of the cavity;

$u$ and $v$ are the acoustic mode numbers;

$U$ and $V$ are the numbers of modes used.

$\varphi_{u v}(x, y)$ is the acoustic pressure mode shape function;

$L_{u v}^{h}$ and $N_{u v}^{h}$ are the constants depending on the boundary conditions.

Consider the boundary conditions at $x=0, a$ and $y=0, b$

$$
\begin{aligned}
& \frac{\partial P^{h}}{\partial x}=0 \text { at } x=0 \text { and } a \\
& \frac{\partial P^{h}}{\partial y}=0 \text { at } y=0 \text { and } b
\end{aligned}
$$

From the above boundary conditions, it can be inferred that the suitable acoustic mode shape is a double cosine function.

$$
\varphi_{u v}(x, y)=\cos \left(\frac{u \pi}{a} x\right) \cos \left(\frac{v \pi}{b} y\right)
$$

From this we can use the following two boundary conditions and the general solution form in Equation (12) to obtain the normalized acoustic pressures at $z=0$ and $c$ (i.e., $P_{c}$ and $P_{0}$ )

$$
\begin{aligned}
& \frac{\partial P^{1}}{\partial z}=-\rho_{a} \frac{\partial^{2} w_{o}^{1}(x, y, t)}{\partial t^{2}} \text { at } z=0 \\
& \frac{\partial P^{h}}{\partial z}=-\rho_{a} \frac{\partial^{2} w_{c}^{h}(x, y, t)}{\partial t^{2}} \text { at } z=c
\end{aligned}
$$

where $\rho_{a}$ is air density; $c$ is cavity depth. Note that there is only the fundamental harmonic component at $z=0$. This is because the flexible wall vibration is linear.

From Equations (12), (16) and (17), the normalized acoustic pressures can be given by

$$
\begin{gathered}
P_{c}=-K_{o}^{1} \bar{B}+\sum_{h=1,3,5 \ldots}^{H} K_{c}^{h} \bar{A}^{h} \\
P_{o}=K_{c}^{1} \bar{B}-\sum_{h=1,3,5 \ldots}^{H} K_{o}^{h} \bar{A}^{h}
\end{gathered}
$$

where $\bar{A}^{h}=$ the $h$ th harmonic component of $\bar{A}$ (i.e., $\bar{A}=\sum_{h=1,3,5}^{H} \bar{A}^{h}$ );

Coupled stiffness at $z=\mathrm{c}, K_{c}^{h}=\sum_{u}^{U} \sum_{v}^{V} \frac{\rho_{a}(h \omega)^{2}}{\mu_{u v}^{h}} \frac{\left(\alpha_{u v}^{m n}\right)^{2}}{\alpha_{u v}^{u \nu} \alpha_{m n}^{m n}} \operatorname{coth}\left(\mu_{u v}^{h} c\right)$;

Coupled stiffness at $z=0, K_{o}^{h}=\sum_{u}^{U} \sum_{v}^{V} \frac{\rho_{a}(h \omega)^{2}}{\mu_{u v}^{h}} \frac{\alpha_{u v}^{m n} \alpha_{u v}^{11}}{\alpha_{u v}^{u v} \alpha_{m n}^{m n}} \frac{1}{\sinh \left(\mu_{u v}^{h} c\right)}$;

Coupling factor between nonlinear panel and cavity modes, $\alpha_{u v}^{m n}=\int_{0}^{b} \int_{0}^{a} \varphi_{u v} \varphi_{m n} d x d y$;

Coupling factor of cavity modes, $\alpha_{u v}^{u v}=\int_{0}^{b} \int_{0}^{a} \varphi_{u v} \varphi_{u v} d x d y$;

Coupling factor of panel modes, $\alpha_{m n}^{m n}=\int_{0}^{b} \int_{0}^{a} \varphi_{m n} \varphi_{m n} d x d y$;

Coupling factor between flexible wall and cavity modes, $\alpha_{u v}^{11}=\int_{0}^{b} \int_{0}^{a} \varphi_{u v} \varphi_{11} d x d y$;

Nonlinear panel mode function, $\varphi_{m n}(x, y)=\sin \left(\frac{m \pi}{a} x\right) \sin \left(\frac{n \pi}{b} y\right)$;

Flexible wall mode shape, $\varphi_{11}(x, y)=\sin \left(\frac{\pi}{a} x\right) \sin \left(\frac{\pi}{b} y\right)$ (i.e., $m=n=1$ ).

This is because the flexible wall is thick, and its fundamental resonant frequency is much higher than that of the thin panel absorber. 


\section{Results and Discussion}

Table 1 shows the material properties, physical dimensions and other input parameters in the numerical cases in this study. Tables 2 and 3 show the mode convergences for the center pressures at $z=0$ and $c$ induced by the nonlinear panel and flexible wall, respectively. In the convergence studies, two harmonic terms are adopted. The center pressures obtained from the 16-mode approach are normalized as one. It can be seen in the center pressures induced by the nonlinear panel that there are more modes required for good convergence if the cavity depth is longer. Generally, the nine acoustic mode approach is good enough for the ratio of a cavity depth to panel width of less than 1 . The center pressure induced by the linearly vibrating flexible wall makes it much easier to find the convergence.

Table 1. Material properties and symmetric panel configurations.

\begin{tabular}{cc}
\hline Panel dimensions & $0.3048 \mathrm{~m} \times 0.3048 \mathrm{~m} \times 1.2192 \mathrm{~mm}$ \\
\hline Young's modulus & $7 \times 10^{10} \mathrm{~N} / \mathrm{m}^{2}$ \\
\hline Material density & $2700 \mathrm{~kg} / \mathrm{m}^{3}$ \\
\hline Poisson's ratio & 0.3 \\
\hline Air density & $1.2 \mathrm{~kg} / \mathrm{m}^{3}$ \\
\hline Sound speed & $340 \mathrm{~m} / \mathrm{s}$ \\
\hline
\end{tabular}

Table 2. Convergence study for the center pressure at $z=c$ induced by the nonlinear symmetric panel.

\begin{tabular}{cccccc}
\hline \multirow{2}{*}{ Acoustic Modes Used } & \multicolumn{5}{c}{ Cavity Depth/Panel width } \\
\cline { 2 - 6 } & $\mathbf{0 . 1}$ & $\mathbf{0 . 2}$ & $\mathbf{0 . 5}$ & $\mathbf{1}$ & $\mathbf{2}$ \\
\hline $\mathrm{u}=\mathrm{v}=0$ & 1.0334 & 1.0454 & 1.1079 & 1.3285 & 0.4452 \\
$\mathrm{u}=\mathrm{v}=0,2$ & 0.9977 & 0.9961 & 0.9895 & 0.9678 & 1.0543 \\
$\mathrm{u}=\mathrm{v}=0,2,4$ & 1.0008 & 1.0016 & 1.0042 & 1.0128 & 0.9784 \\
$\mathrm{u}=\mathrm{v}=0,2,4,6$ & 1.0000 & 1.0000 & 1.0000 & 1.0000 & 1.0000 \\
\hline
\end{tabular}

Table 3. Convergence study for the center pressure at $z=0$ induced by the nonlinear symmetric panel.

\begin{tabular}{cccccc}
\hline \multirow{2}{*}{ Acoustic Modes Used } & \multicolumn{5}{c}{ Cavity Depth/Panel width } \\
\cline { 2 - 6 } & $\mathbf{0 . 1}$ & $\mathbf{0 . 2}$ & $\mathbf{0 . 5}$ & $\mathbf{1}$ & $\mathbf{2}$ \\
\hline $\mathrm{u}=\mathrm{v}=0$ & 1.0279 & 1.0227 & 1.0074 & 1.0006 & 1.0000 \\
$\mathrm{u}=\mathrm{v}=0,2$ & 0.9987 & 0.9993 & 1.0000 & 1.0000 & 1.0000 \\
$\mathrm{u}=\mathrm{v}=0,2,4$ & 1.0002 & 1.0001 & 1.0000 & 1.0000 & 1.0000 \\
$\mathrm{u}=\mathrm{v}=0,2,4,6$ & 1.0000 & 1.0000 & 1.0000 & 1.0000 & 1.0000 \\
\hline
\end{tabular}

Figure 2 shows the amplitude ratio of the nonlinear symmetric panel plotted against the frequency ratio for various panel resonances. Note that the wall is rigid. Obviously, the first three frequency ratios are monotonically increasing with the amplitude ratio. The first resonant frequency ratio (the solid line) is compared with a set of circles obtained from [20]. The first resonant frequency ratios obtained from the proposed method and the finite element method agree reasonably well with each other. When the amplitude ratio = 0 , the higher mode resonant frequency ratios (i.e., $(1,3)$ and $(3,3)$ modes) are close to one, and the first resonant frequency ratio is much higher. It is implied that the higher mode resonant frequencies are not significantly affected by the air cavity stiffness. The slope of the solid line is deeper than those of the dashed and dotted lines, which represent the $(1,3)$ and $(3,3)$ mode frequency ratios. This implies that the higher mode resonant frequency ratios are more sensitive to the vibration amplitude. Figure 3 shows the amplitude ratio plotted against the first resonant frequency ratio for various thickness ratios (i.e., the ratio of wall thickness to nonlinear panel thickness, $\tau_{o} / \tau_{c}$ ). Note that the nonlinear symmetric 
panel thickness is kept unchanged. When the wall is thicker, the overall cavity stiffness is stronger. This explains why the line of $\tau_{o} / \tau_{c}=8$ is the farthest to the right. This implies that the resonant frequency and the stiffness in this case are the highest among them. Interestingly, all these lines look quite parallel to each other. For $A_{\text {in }} / \tau_{c}=0$, the frequency ratio difference between the cases of $\tau_{o} / \tau_{c}=2.5$ and 8 is about $7.2 \%$; for $A_{\text {in }} / \tau_{c}=1.4$, the frequency ratio difference between the cases of $\tau_{o} / \tau_{c}=2.5 ; 8$ is also about $7.2 \%$.

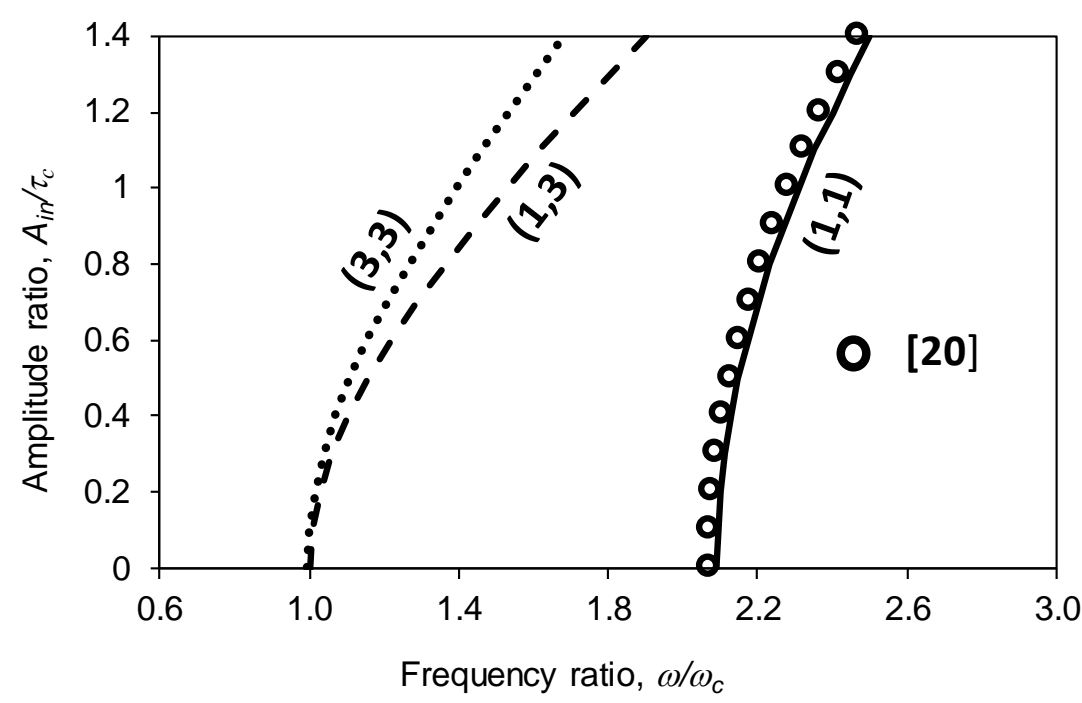

Figure 2. Amplitude ratio vs. frequency ratio for various resonances.

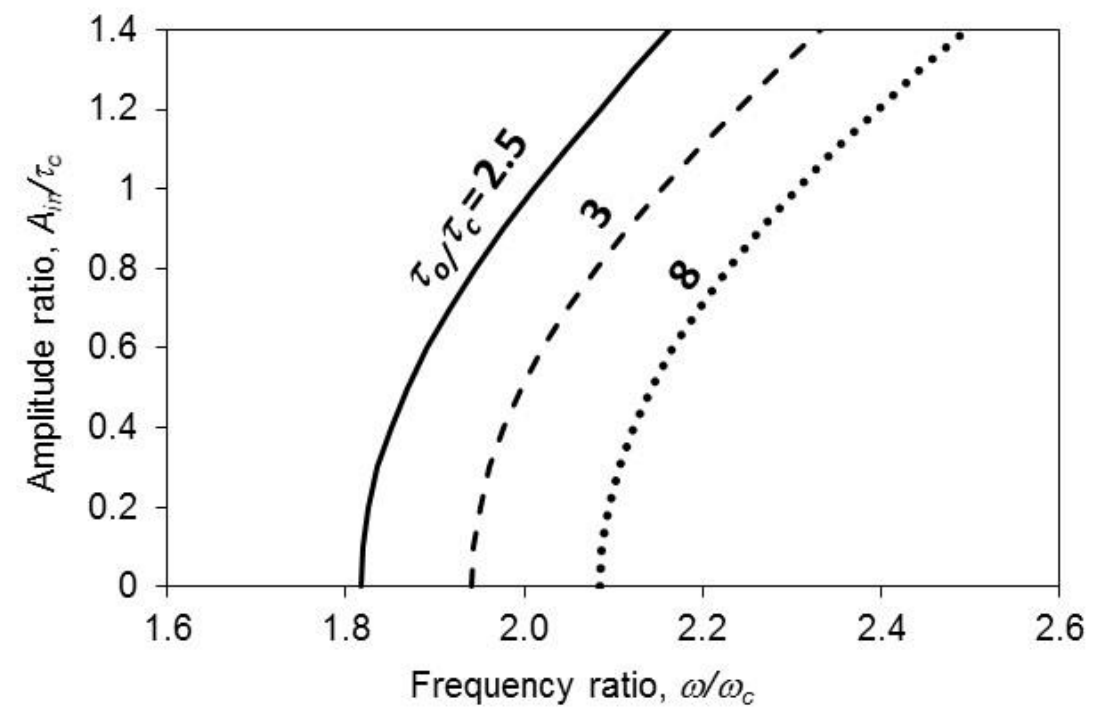

Figure 3. Amplitude ratio vs. first resonant frequency ratio for various thickness ratios.

Figure 4 shows the nonlinear panel amplitude ratio plotted against the flexible wall amplitude ratio for various thickness ratios. All of the ratios monotonically increase. The line of $\tau_{o} / \tau_{c}=8$ is almost vertical. This implies that the wall vibration amplitude is inert to the nonlinear vibration amplitude. Comparatively, the wall stiffness is much stronger than the nonlinear panel stiffness, and it can be considered as nearly rigid. Hence, the ratio of wall amplitude to nonlinear panel amplitude is very small. An example of this is the line of $\tau_{o} / \tau_{c}=3$, when $A_{i n} / \tau_{c}=1, B_{o} / \tau_{o}=0.0691$. All lines in Figure 4 are nearly linear, particularly for the cases with a high $\tau_{0} / \tau_{c}$ ratio. In the cases of $\tau_{o} / \tau_{c}=3$ and 2.5, the lines are slightly curved when $A_{i n} / \tau_{c}>1$. Figure 5 shows the first resonant frequency ratio plotted against the thickness ratio for various amplitude ratios. The frequency ratio in the case of the highest $A_{i n} / \tau_{c}$ is always higher than those of the other two cases because the 
highest ratio of $A_{\text {in }} / \tau_{c}$ represents the highest stiffness. When $\tau_{o} / \tau_{c}>5$, the three lines are almost horizontal. This implies that the wall is stiff when the ratio of $\tau_{o} / \tau_{c}=5$. There is not much change even after the ratio of $\tau_{o} / \tau_{c}$ further increases. Figure 6 shows the first resonant frequency ratio plotted against the thickness ratio for various cavity depths. The frequency ratio in the case of the shortest cavity depth is always higher than those in the other two cases. This is because the cavity with the shortest depth is the stiffest. Similar to those in Figure 5, the three lines in Figure 6 are almost horizontal when $\tau_{o} / \tau_{c}>5$. Unlike those in Figure 5, the lines of cavity depths $=0.1524 \mathrm{~m}$ and $0.1016 \mathrm{~m}$ are insensitive to the ratio of $\tau_{o} / \tau$ for almost the entire range. Only the line of cavity depth $=0.0508 \mathrm{~m}$ is sensitive to the ratio of $\tau_{o} / \tau$ when it is less than 3. Figure 7 shows the center pressure ratio plotted against the cavity depth ratio for various amplitude ratios. The three lines are monotonically decreasing. When the cavity depth is short (e.g., $c=0.1 \mathrm{~m}$ ), the three center pressure ratios are almost the same. When the cavity depth increases in length, the three lines deviate from each other. In the linear case and the most nonlinear case (i.e., the amplitude ratios $=0$ and 1.2), the pressure ratios are always the highest and lowest for the entire range, respectively. The higher pressure ratio implies that the pressure at $z=c$ transmitted to $z=0$ is smaller (lower transmission loss).

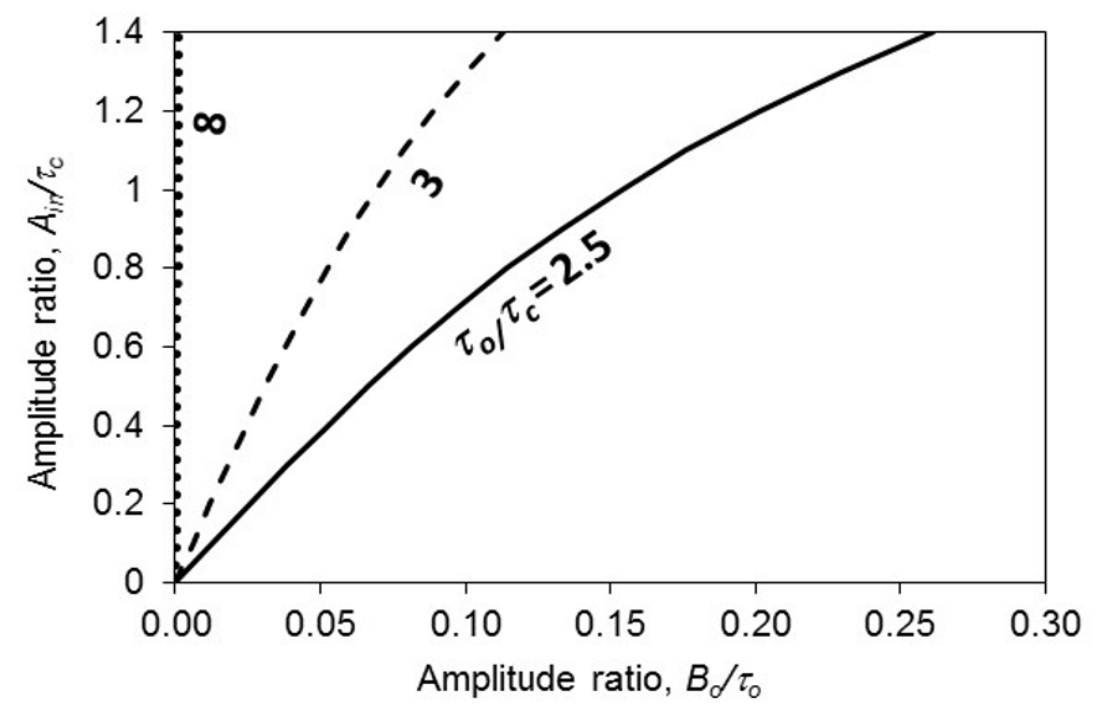

Figure 4. Nonlinear panel amplitude ratio vs. flexible wall amplitude ratio for various thickness ratios.

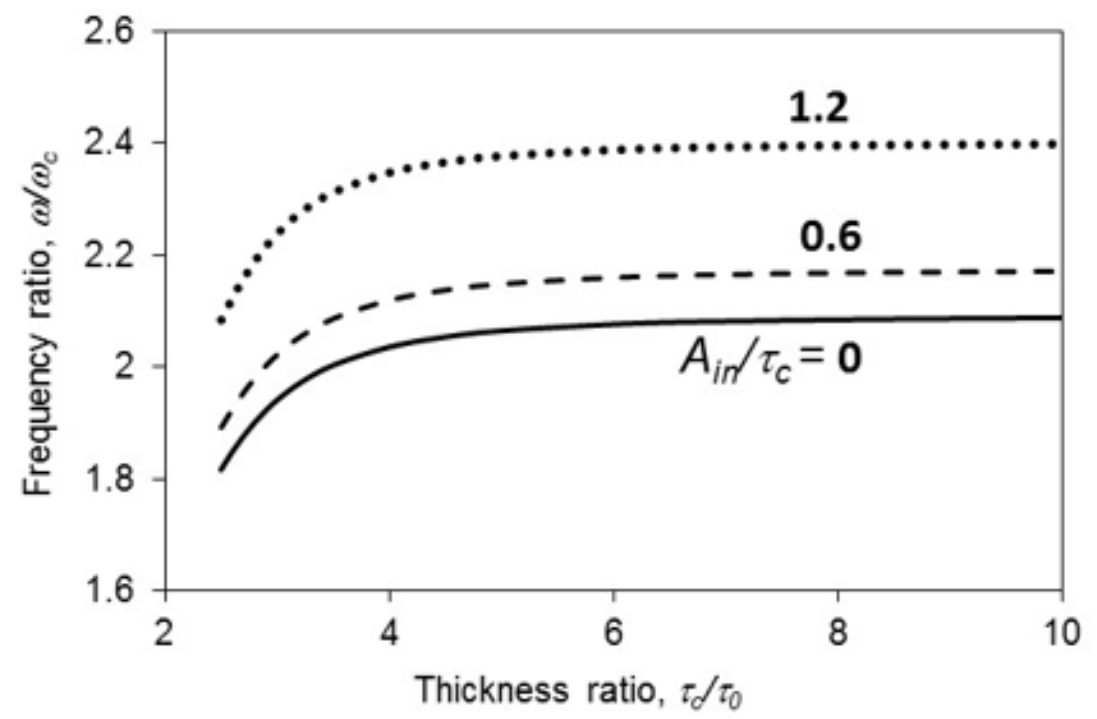

Figure 5. First resonant frequency ratio vs thickness ratio for various amplitude ratios. 


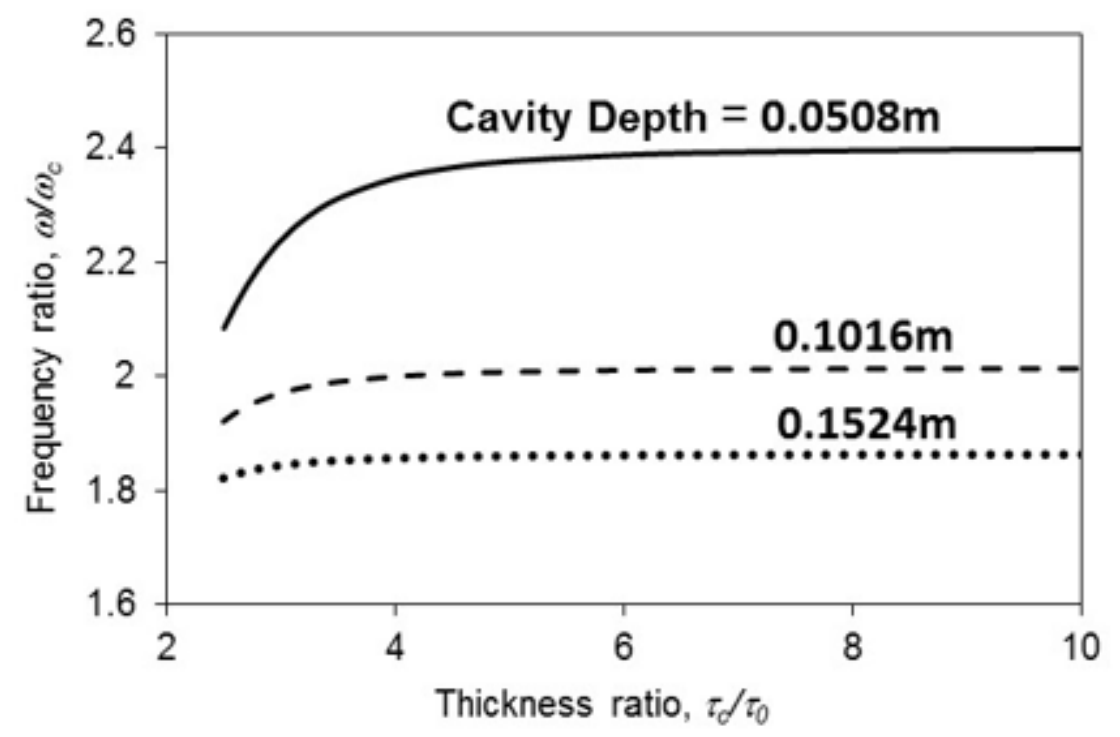

Figure 6. First resonant frequency ratio vs. thickness ratio for various cavity depths.

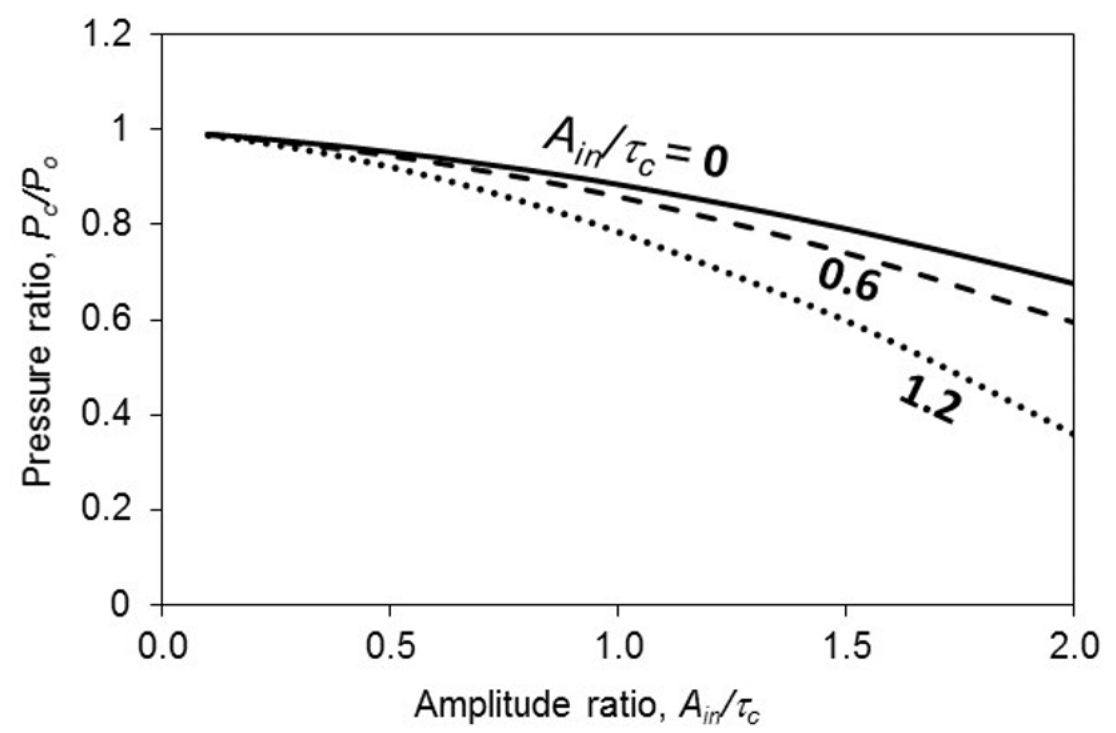

Figure 7. Center pressure ratio vs. cavity depth ratio for various amplitude ratios.

Figure 8 shows the center pressure ratio plotted against the amplitude ratio for various cavity depth ratios. Unlike those in Figure 7, only the line with the longest cavity depth in Figure 8 is monotonically decreasing. The other two lines are almost horizontal, or the two center pressure ratios are inert to the amplitude ratio. This implies that the ratio of the pressure at $z=c$ transmitted to $z=0$ is almost constant. In the case of the longest cavity depth, the cavity stiffness is the weakest so that overall cavity stiffness is sensitive to nonlinear stiffness, and it can significantly affect the pressure transmission ratio. 


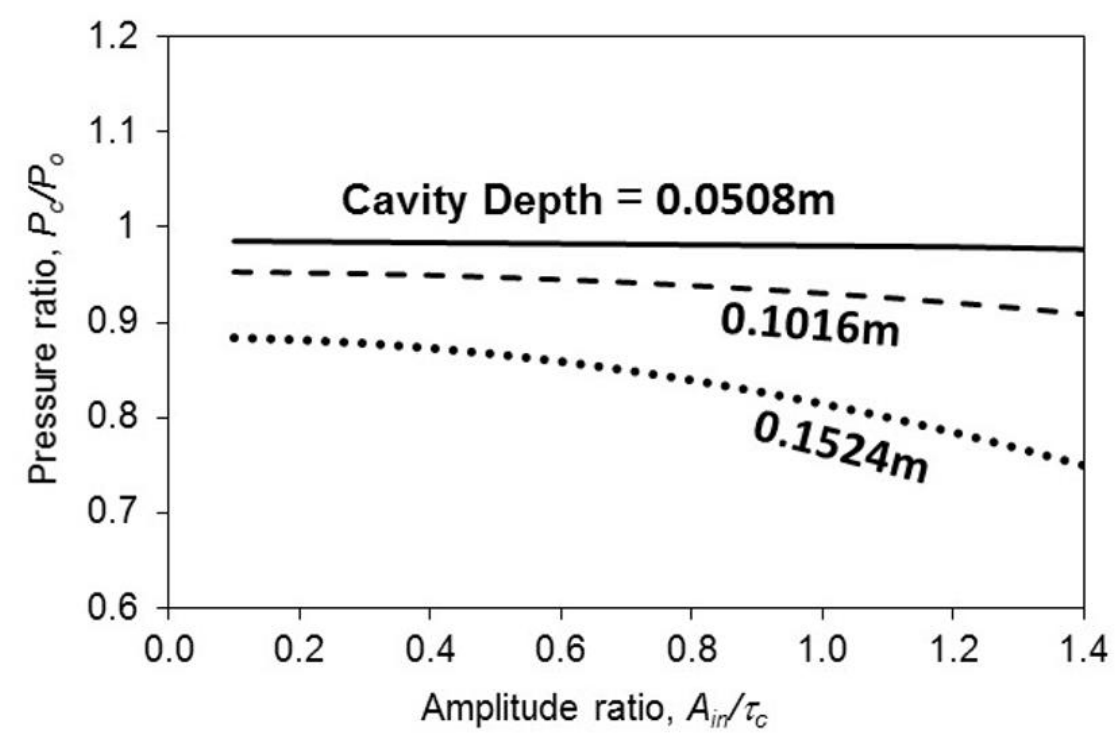

Figure 8. Center pressure ratio vs. amplitude ratio for various cavity depth ratios.

\section{Conclusions}

This study has presented the formulations for a nonlinear symmetric panel absorber mounted on a flexible wall, and has developed the frequency-amplitude relationship. This structural-acoustic problem considers the nonlinear and linear vibrations coupled with the multi-mode acoustic pressures within a cavity. The results of nonlinear resonant frequency and acoustic pressure are obtained using the weighted residual method and elliptic integral solution form. The results obtained from the proposed method and finite element method agree reasonably well with each other. The numerical simulations show that (1) the flexible wall can soften the overall cavity stiffness to make the resonant frequency lower, even though the wall vibration amplitude is much smaller than the nonlinear panel vibration amplitude; (2) when the thickness ratio of $\tau_{o} / \tau_{c}=5$, the wall can be considered as very stiff or rigid. The frequency ratio changes very little even though the ratio of $\tau_{o} / \tau_{c}$ further increases; and (3) when the cavity depth is long (e.g., $0.3024 \mathrm{~m}$ ), the cavity stiffness is weak so that it is sensitive to the nonlinear stiffness induced by the large amplitude vibrations, and able to affect the pressure transmission ratio significantly.

As aforementioned, when the vibration amplitude of a panel is larger, the resonant frequency or panel stiffness is higher. In a typical linear design, it is assumed that the panel stiffness is constant and does not depend on the vibration amplitude. Thus, it would result in overdesign, especially for cases of large excitation or large vibration amplitude. This frequency-amplitude work can be a reference for a nonlinear panel absorber design.

Author Contributions: Data curation, Y.-Y.L.; formal analysis, Y.-Y.L.; investigation, Y.-Y.L.; methodology, Y.-Y.L.; writing and editing, Y.-Y.L. The sole author has read and agreed to the published version of the manuscript.

Funding: This research received no external funding.

Institutional Review Board Statement: Not applicable.

Informed Consent Statement: Not applicable.

Data Availability Statement: Not applicable.

Conflicts of Interest: The author declares no conflict of interest.

\section{References}

1. Ford, R.D.; Mccormick, M.A. Panel sound absorbers. J. Sound Vib. 1969, 10, 411-423. [CrossRef]

2. Lyon, R.H. Noise reduction of rectangular enclosures with one flexible wall. J. Acoust. Soc. Am. 1963, 35, 1791-1797. [CrossRef]

3. Pretlove, A.J. Free vibrations of a rectangular panel backed by a closed rectangular cavity. J. Sound Vib. 1965, 2, 197-209. [CrossRef] 
4. Jackson, R.S. Some aspects of the performance of acoustic hoods. J. Sound Vib. 1966, 3, 82-94. [CrossRef]

5. Frommhold, W.; Fuchs, H.V.; Sheng, S. Acoustic performance of membrane absorbers. J. Sound Vib. 1994, 170, 621-636. [CrossRef]

6. Chiang, Y.K.; Choy, Y.S. Acoustic behaviors of the micro-perforated panel absorber array in nonlinear regime under moderate acoustic pressure excitation. J. Acoust. Soc. Am. 2018, 143, 538-549. [CrossRef]

7. Pan, J.; Elliott, S.J.; Baek, K.H. Analysis of low frequency acoustic response in a damped rectangular enclosure. J. Sound Vib. 1999, 223, 543-566. [CrossRef]

8. Nehete, D.V.; Modak, S.V.; Gupta, K. Structural FE model updating of cavity systems incorporating vibro-acoustic coupling. Mech. Syst. Signal Process. 2015, 50-51, 362-379. [CrossRef]

9. He, Z.C.; Liu, G.R.; Zhong, Z.H.; Cui, X.Y.; Zhang, G.Y.; Cheng, A.G. A coupled edge-/face-based smoothed finite element method for structural-acoustic problems. Appl. Acoust. 2010, 71, 955-964. [CrossRef]

10. Lee, Y.Y. Sound and vibration analysis of a nonlinear panel absorber mounted on an enclosure panel using the multi-level residue harmonic balance method. Appl. Math. Model. 2015, 39, 4995-5010. [CrossRef]

11. Lee, Y.Y. Natural frequency analysis of two nonlinear panels coupled with a cavity using the approximate elliptic integral solution and the method of harmonic residual minimization. Discret. Dyn. Nat. Soc. 2015, 2015, 939502. [CrossRef]

12. Sadri, M.; Younesian, D. Nonlinear free vibration analysis of a plate-cavity system. Thin-Walled Struct. 2014, 74, 191-200. [CrossRef]

13. Elias-Zuniga, A.; Palacios-Pineda, L.M.; Jimenez-Cedeno, I.H.; Martinez-Romero, O.; Olvera-Trejo, D. Enhanced He's frequencyamplitude formulation for nonlinear oscillators. Results Phys. 2020, 19, 103626. [CrossRef]

14. Wei, Y.; Vaicaitis, R. Nonlinear models for double-wall systems for vibrations and noise control. J. Aircr. 1997, 34, 802-810. [CrossRef]

15. Yang, C.J. Approximate Super- and Sub-harmonic Response of a Multi-DOFs System with Local Cubic Nonlinearities under Resonance. J. Appl. Math. 2012, 531480.

16. Zheng, Z.L.; Liu, C.J.; He, X.T.; Chen, S.L. Free Vibration Analysis of Rectangular Orthotropic Membranes in Large Deflection. Math. Probl. Eng. 2009, 634362.

17. Leung, A.Y.T.; Guo, Z.J. Residue harmonic balance approach to limit cycles of non-liner jerk equations. Int. J. Non-Linear Mech 2011, 46, 898-906. [CrossRef]

18. Rahman, M.S.; Hasan, A.S.M.Z. Modified harmonic balance method for the solution of nonlinear jerk equations. Results Phys. 2018, 8, 893-897. [CrossRef]

19. Sze, K.Y.; Chen, S.H.; Huang, J.L. The incremental harmonic balance method for nonlinear vibration of axially moving beams. J. Sound Vib. 2005, 281, 611-626. [CrossRef]

20. Lee, Y.Y. Structural-acoustic coupling effect on the nonlinear natural frequency of a rectangular box with one flexible plate. Appl. Acoust. 2002, 63, 1157-1175. [CrossRef] 